\title{
Finite-Frequency SKS Splitting: Measurement and Sensitivity Kernels
}

\author{
by Anne Sieminski, Hanneke Paulssen, Jeannot Trampert, and Jeroen Tromp
}

\begin{abstract}
Splitting of $S K S$ waves caused by anisotropy may be analyzed by measuring the splitting intensity, i.e., the amplitude of the transverse signal relative to the radial signal in the $S K S$ time window. This quantity is simply related to structural parameters. Extending the widely used cross-correlation method for measuring travel-time anomalies to anisotropic problems, we propose to measure the $S K S$ splitting intensity by a robust cross-correlation method that can be automated to build large high-quality datasets. For weak anisotropy, the $S K S$-splitting intensity is retrieved by cross-correlating the radial signal with the sum of the radial and transverse signals. The cross-correlation method is validated based upon a set of Californian seismograms. We investigate the sensitivity of the $S K S$-splitting intensity to general anisotropy in the mantle based upon a numerical technique (the adjoint spectralelement method) considering the full physics of wave propagation. The computations reveal a sensitivity remarkably focused on a small number of elastic parameters and on a small region of the upper mantle. These fundamental properties and the practical advantages of the measurement make the cross-correlation $S K S$-splitting intensity particularly well adapted for finite-frequency imaging of upper-mantle anisotropy.
\end{abstract}

\section{Introduction}

Information from shear-wave splitting is often used to constrain deformation in the Earth. Such deformation can preferentially orient anisotropic minerals of the mantle over large scales, making the elastic structure anisotropic for seismic-wave propagation (e.g., Kaminski and Ribe, 2002). A characteristic property of wave propagation in anisotropic media is shear-wave splitting. In such a medium, two orthogonally polarized $S$ waves propagate with different speeds (e.g., Babuška and Cara, 1991). Analyzing shear-wave splitting thus gives information on the anisotropy of the material, which can then be interpreted in terms of deformation with the help of mineral physics. A common way of analyzing shear-wave splitting is to measure the time shift between the fast and slow $S$ waves and the polarization azimuth of the fast wave. The time shift is proportional to the magnitude of anisotropy and the length of the path in the anisotropic structure. For a single homogeneous anisotropic layer with hexagonal symmetry and a horizontal symmetry axis, assuming vertical incidence, the polarization of the fast $S$ wave is aligned with the orientation of the fast symmetry axis. Measuring the polarization of the fast wave requires that we know the polarization of the unsplit $S$ wave in the isotropic reference model. $S K S$ and $S K K S$ waves are thus very convenient for shear-wave splitting measurements, and most of the present shear-wave splitting datasets involve these waves. $S K S$ and $S K K S$ waves are core phases. When entering the liquid outer core, $S$ waves are converted into $P$ waves. This conversion makes them emerge out of the core as ra- dially polarized $S V$ waves. Because they are teleseismic phases, their propagation is nearly vertical from the coremantle boundary (CMB) to the surface. In an isotropic model, they mainly appear on the radial component at the receivers. However, if they encounter an anisotropic structure between the CMB and the surface, they are split and some signal is observed on the transverse component.

The $S K S$-splitting parameters (time shift and fast polarization) only give direct insight into the anisotropic properties of the Earth in the case of a single homogeneous layer with hexagonal symmetry and a horizontal axis. For more complex anisotropy (i.e., multiple layers, laterally varying anisotropy, a tilted symmetry axis, or lower symmetries), the measured time shift and fast polarization are only apparent parameters without a direct correspondence to the characteristics of the structure, and additional analysis is required (Silver and Savage, 1994; Levin et al., 1999; Hartog and Schwarz, 2000, 2001). Most studies to date have been based on an asymptotic description of wave propagation (i.e., ray theory). In the asymptotic description, body waves are evenly sensitive to perturbations of structural parameters along the geometrical ray path. Travel-time anomalies are thus an integrated measure of the perturbations in wave speed along the ray path. Similarly, the $S K S$-splitting time shift reflects the properties of anisotropy integrated from the CMB to the surface, while the fast polarization corresponds to the last anisotropic layer seen by the wave (Chen and Tromp, 2007). In this description, the shear-wave splitting measurements 
provide good lateral resolution but poor vertical resolution. Shear-wave splitting measurements, however, are finitefrequency data. The commonly analyzed waveforms have a dominant period varying from $\sim 3 \mathrm{sec}$ (Hartog and Schwarz, 2000, 2001; Polet and Kanamori, 2002) to $\sim 10 \mathrm{sec}$ (Long and van der Hilst, 2005; Lev et al., 2006). A Fresnel-zone description gives some indication about the location in depth of the detected anisotropy. In such a Fresnel-zone description, body waves are sensitive to the structure over a finite-size frequency-dependent volume around the ray path. By comparing $S K S$-splitting measurements at neighboring stations, it is possible to infer upper and lower limits for the location of anisotropy (Alsina and Snieder, 1995).

Seismic tomography recently has moved from an asymptotic description of wave propagation to finitefrequency theories (e.g., Montelli et al., 2004; Chen et al., 2007). This has been motivated by the firm belief that significant progress in imaging the Earth's structure requires accommodation of finite-frequency aspects of wave propagation. This change was complemented by the extensive use of the cross-correlation method to measure travel-time anomalies. Cross-correlation travel-time anomalies are intrinsically finite-frequency data that should not be analyzed with asymptotic theory. The cross-correlation method has several practical advantages. It has allowed seismologists to more quickly build large and high-quality datasets than feasible through manual picking (Woodward and Masters, 1991; Su and Dziewonski, 1992; Ritsema and van Heijst, 2002). $S K S$-splitting observables should similarly be improved for higher-resolution imaging of anisotropy, emphasizing large datasets and finite-frequency theories. We cannot apply the Born formalism of Dahlen et al. (2000) directly to the usual two-parameter description of $S K S$ splitting, because the time shift and the fast polarization are not simply linked to structural parameters. Finite-frequency $S K S$-splitting imaging therefore requires the definition of other observables that capture the frequency dependence of wave propagation and are simply related to perturbations of structural parameters. The splitting intensity introduced by Chevrot (2000) fully satisfies these requirements. This observable measures the perturbation of the transverse signal relative to the radial signal. Based on a Born-scattering plane-wave formalism, Chevrot (2006) subsequently developed a finite-frequency vectorial tomography to image hexagonal anisotropy using an $S K S$-splitting intensity dataset. An important result from Chevrot's (2006) synthetic tests is that good vertical resolution can be achieved when the frequency dependence of $S K S$ splitting is considered. We propose here to measure the $S K S$ splitting intensity by a stable cross-correlation method. Similar to travel-time measurements, measuring the $S K S$-splitting intensity by cross correlation can be easily automated to obtain large datasets.

After briefly reviewing the classical $S K S$-splitting observables and measurement techniques, we present and validate the cross-correlation method with a northern California dataset in the section entitled Measurements. In the section entitled Sensitivity, we explore how the $S K S$-splitting intensity sees general anisotropy by investigating its sensitivity to perturbations of 21 elastic parameters with the adjoint spectral-element method (Tromp et al., 2005; Liu and Tromp, 2006; Sieminski et al., 2007a, 2007b). This numerical method accounts for the full physics of seismic-wave propagation and provides the sensitivity to first-order perturbations. The results highlight interesting properties of the $S K S$-splitting intensity for imaging mantle anisotropy.

\section{Measurements}

\section{The Two-Parameter Approach}

Traditional shear-wave splitting studies measure the time shift $\Delta T$ between the fast and slow $S$ waves and the polarization azimuth of the fast wave $\phi_{f}$. The methods to measure these parameters usually assume that only one single split is visible at the receiver. In this case, the $S$-wave signal d observed at the receiver on the radial and transverse components (Silver and Chan, 1991; Chevrot et al., 2004) can be obtained from the isotropic reference $S$-wave signal $\mathbf{s}$, such that, in the frequency domain,

$$
\mathbf{d}=R^{-1} D R \mathbf{s} .
$$

The matrix $R$ expresses the rotation from the radialtransverse coordinates to the fast-slow coordinates. It depends on the fast polarization $\phi_{f}$. The matrix $D$ is a time-shift operator controlled by the time shift $\Delta T$. Measuring the splitting parameters $\phi_{f}$ and $\Delta T$ consists of finding the inverse operator of $R^{-1} D R$ that cancels the observed splitting. For $S K S$ waves, a search for the parameters $\left(\phi_{f}, \Delta T\right)$ is made by minimizing the energy of the corrected transverse signal (Vinnik et al., 1989; Silver and Chan, 1988, 1991). For general $S$ waves, the criterium is to maximize the linearity of the corrected $S$-wave particle motion (Silver and Chan, 1991) or the similarity in the pulse shape of the corrected fast and slow signals (Levin et al., 1999). Uncertainties in the measurements are estimated by an F-test (Silver and Chan, 1991). These different techniques give similar results for simple anisotropy, but significant discrepancies have been observed in complex structures like subduction zones (Long and van der Hilst, 2005) or when one is close to a null measurement (Wüstefeld and Bokelmann, 2007). A null measurement occurs when there is no apparent splitting. For $S K S$ waves, this happens for a very weak transverse signal. It may be an indication of no significant anisotropy or of a fast axis parallel or orthogonal to the reference polarization. In this case, the measurements of the splitting parameters are not stable.

Figure 1 shows an example of this traditional analysis of $S K S$ splitting for seismograms selected from the northern California dataset of Hartog and Schwartz (2000, 2001). We apply the energy-minimization technique of Silver and Chan (1991) to the signals filtered at different periods. The uncertainties are estimated with an F-test. In Figure 1, we show the seismograms recorded at station YBH for a deep New Britain 
dominant period $\sim 8 \mathrm{~s}$

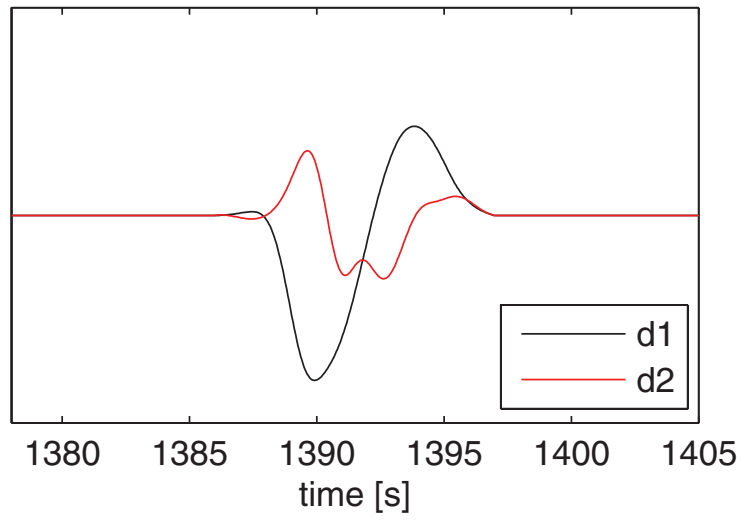

corrected signals with $\phi_{\mathrm{f}}=53^{\circ}$ and $\Delta \mathrm{T}=1.15 \mathrm{~s}$
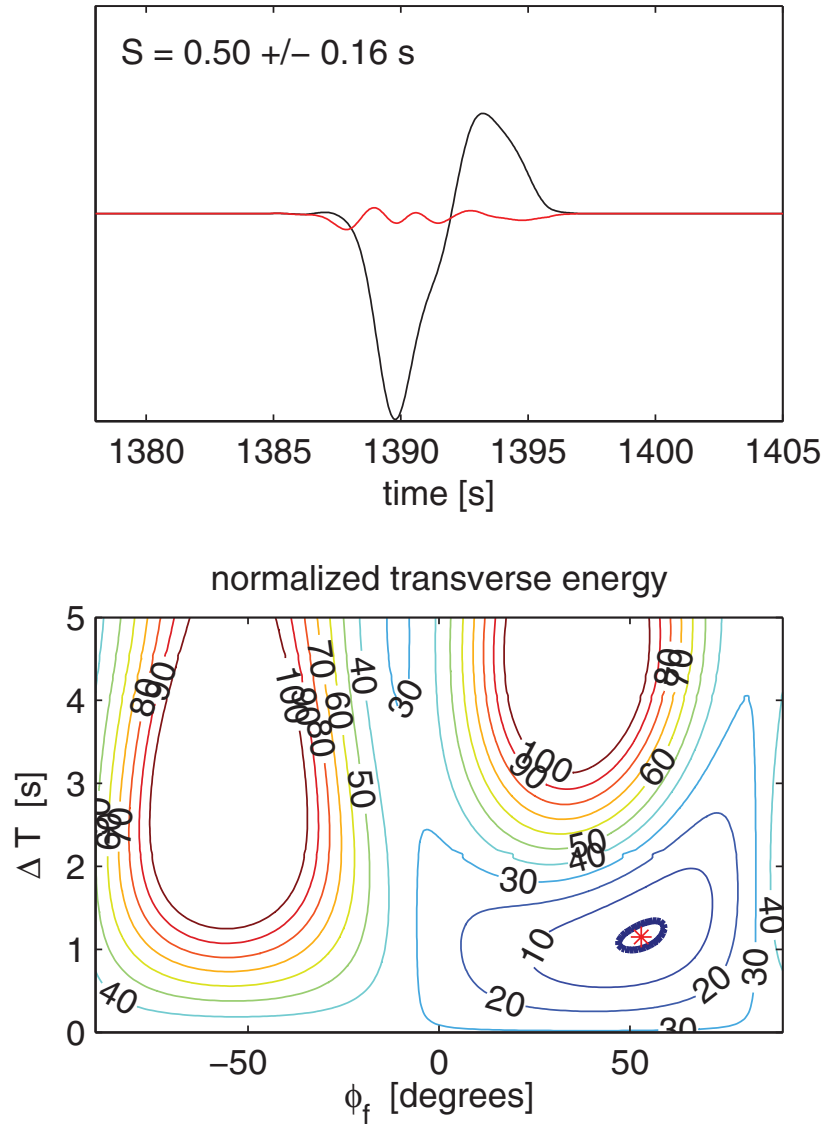

dominant period $\sim 12 \mathrm{~s}$

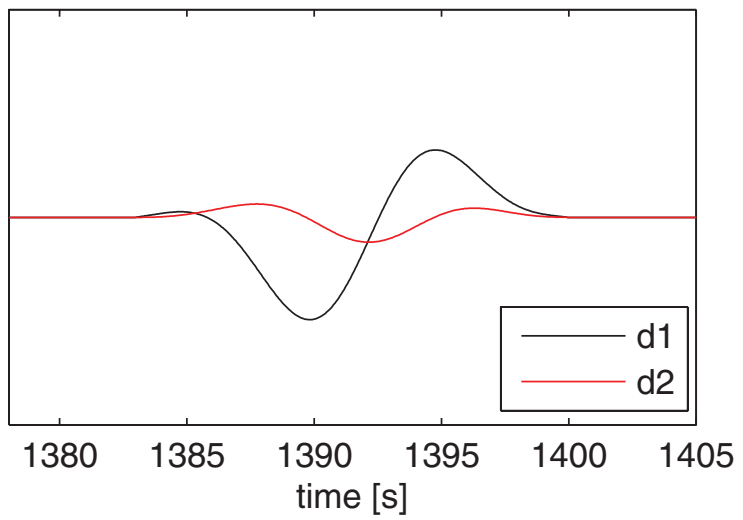

corrected signals with $\phi_{\mathrm{f}}=31^{\circ}$ and $\Delta \mathrm{T}=0.85 \mathrm{~s}$

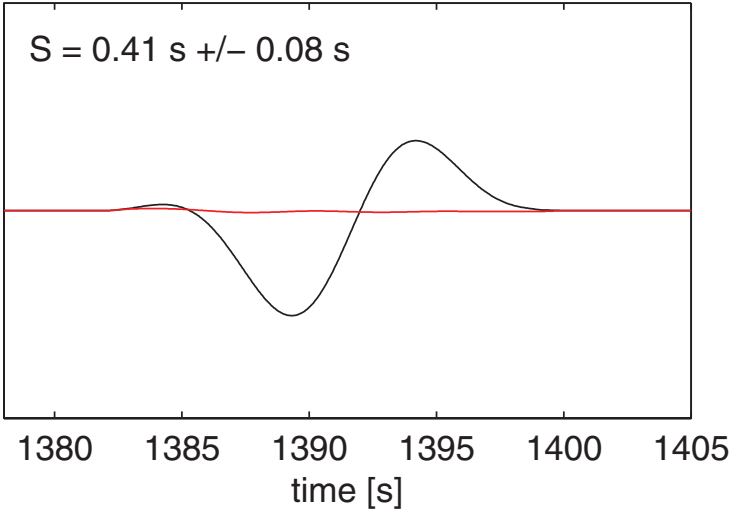

normalized transverse energy

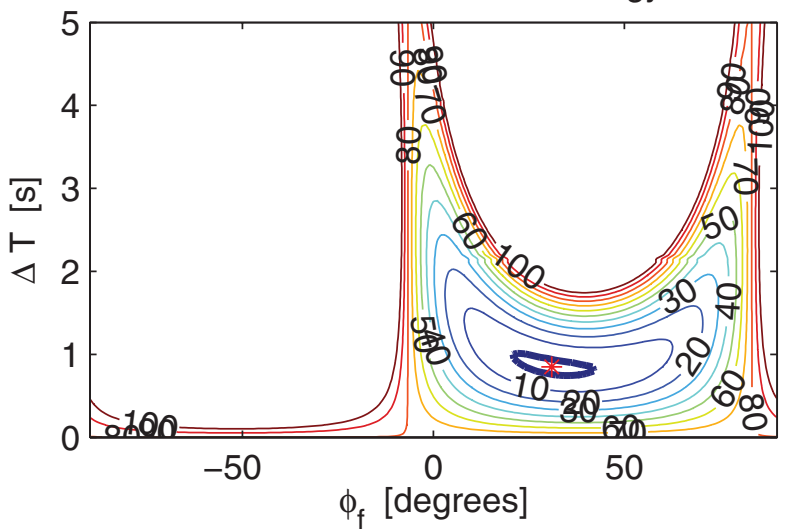

Figure 1. Illustration of the traditional two-parameter approach to analyze $S K S$ splitting. We search for the parameters $\left(\phi_{f}, \Delta T\right)$ minimizing the energy of the transverse component. The top panels show the observed radial $\left(d_{1}\right)$ and transverse signals $\left(d_{2}\right)$; the middle panels show the corrected radial and transverse signals. The signals are time-windowed with a Welch taper centered on the $S K S$ arrival and filtered with an acausal fourth-order band-pass Butterworth filter. The upper period is $100 \mathrm{sec}$, although most of the $S K S$ signal is contained in periods lower than $25 \mathrm{sec}$. The lower period is $1 \mathrm{sec}$ in the left-hand panels and $8 \mathrm{sec}$ in the right-hand panels. The dominant period of the signals is about 8 and $12 \mathrm{sec}$, respectively. The bottom panels are the contours of the transverse energy normalized by the minimum energy as a function of the fast polarization $\phi_{f}$ and the time shift $\Delta T$. The heavy blue contours outline the $95 \%$ confidence region obtained from an F-test and the red star indicates the energy minimum. The splitting intensity $S=-\frac{1}{2} \Delta T \sin 2\left(\phi_{f}-\phi_{0}\right)$ is calculated from the measured values of $\Delta T$ and $\phi_{f}$. The signals are recorded at station YBH of the BDSN for a deep New Britain event (140-km depth) located at an epicentral distance of $90^{\circ}$ (Table 1). 
event band-pass filtered such that the dominant period of the signals is $\sim 8$ or $\sim 12 \mathrm{sec}$. The difference between the results at these two periods reflects the frequency dependence of $S K S$ splitting. It is not the same region of the Earth that is sampled at these different periods. Note that for this region, the splitting parameters at most of the stations vary with the back azimuth of the event. This has been observed in many other places and cannot be explained by laterally varying hexagonal anisotropy with a horizontal symmetry axis (Hartog and Schwartz, 2000, 2001).

For general anisotropy, the relations between the splitting parameters $\Delta T$ and $\phi_{f}$ and the structural parameters are not simple. In an asymptotic description, they are not linearly linked to perturbations of the elastic parameters (Chen and Tromp, 2007). This theoretical difficulty, in addition to the instabilities observed for near-null measurements and the time-consuming grid search, motivates the definition of another description of $S K S$ splitting that is better adapted to imaging.

\section{The Splitting Intensity}

Developing equation (1) in the time domain for $S K S$ (or $S K K S$ ) waves leads to

$$
\begin{aligned}
& d_{1}(t)=s_{1}\left(t-\delta T_{f}\right) \cos ^{2} \psi+s_{1}\left(t-\delta T_{s}\right) \sin ^{2} \psi, \\
& d_{2}(t)=\left[s_{1}\left(t-\delta T_{f}\right)-s_{1}\left(t-\delta T_{s}\right)\right] \sin \psi \cos \psi,
\end{aligned}
$$

where $t$ is the time, $d_{1,2}$ the observed radial and transverse $S K S$ signals, and $s_{1}$ the reference isotropic radial $S K S$ signal (the reference isotropic transverse signal $s_{2}$ is zero). The quantities $\delta T_{f}$ and $\delta T_{s}$ are the time shifts of the fast and slow split $S$ waves, respectively, and we have $\delta T_{s}-\delta T_{f}=\Delta T$. Throughout the article we use the convention of a positive time shift for a delay. The angle $\psi$ is the difference $\phi_{f}-\phi_{0}$, where $\phi_{0}$ is the polarization azimuth of the refer-

\section{dominant period $\sim 8 \mathrm{~s}$}

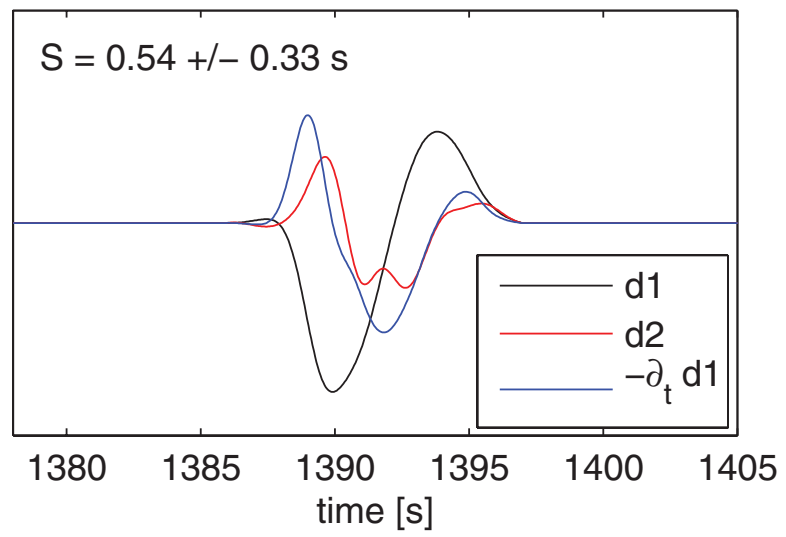

ence $S K S$ wave. For weak anisotropy, the time shifts are small relative to the dominant period of $s_{1}$, and equations (2) and (3) become to first order

$$
\begin{aligned}
d_{1}(t) & =s_{1}(t)-\delta T_{f} \cos ^{2} \psi \dot{s}_{1}(t)-\delta T_{s} \sin ^{2} \psi \dot{s}_{1}(t) \\
& =s_{1}(t)+\delta s_{1}(t), \\
d_{2}(t) & =\frac{1}{2} \Delta T \sin 2 \psi \dot{s}_{1}(t)=\delta s_{2}(t),
\end{aligned}
$$

where the signal $\dot{s}$ is the time derivative of $s$ and $\delta s_{1}(t)=$ $-\delta T_{f} \cos ^{2} \psi \dot{s}_{1}(t)-\delta T_{s} \sin ^{2} \psi \dot{s}_{1}(t)$. Considering weak anisotropy (i.e., small time shifts and a small $\Delta T$ ) implies that $\delta s_{1,2} \ll s_{1}$.

Writing $d_{2}$ as the function of $d_{1}$ such that

$$
d_{2}(t) \simeq \frac{1}{2} \Delta T \sin 2 \psi \dot{d}_{1}(t)
$$

is to first order equivalent to equation (5) for weak anisotropy. $S K S$-splitting studies are commonly based upon equation (6) instead of equation (5) (Vinnik et al., 1989; Levin et al., 1999; Chevrot, 2000). Equation (5) or (6) describes an interesting property of the observed transverse signal for weak anisotropy. This signal is the time derivative of the radial signal (Fig. 2) scaled by the quantity $\frac{1}{2} \Delta T \sin 2 \psi$. Chevrot (2000) proposed to use this property to characterize $S K S$ splitting. He introduced the splitting intensity $S$ as the normalized projection of the transverse signal onto the time derivative of the radial signal,

$$
S=-\frac{\int \dot{d}_{1}(t) d_{2}(t) \mathrm{d} t}{\int \dot{d}_{1}^{2}(t) \mathrm{d} t}
$$

where the integrals are over the $S K S$ time window. Note that the definition we give here is slightly different from

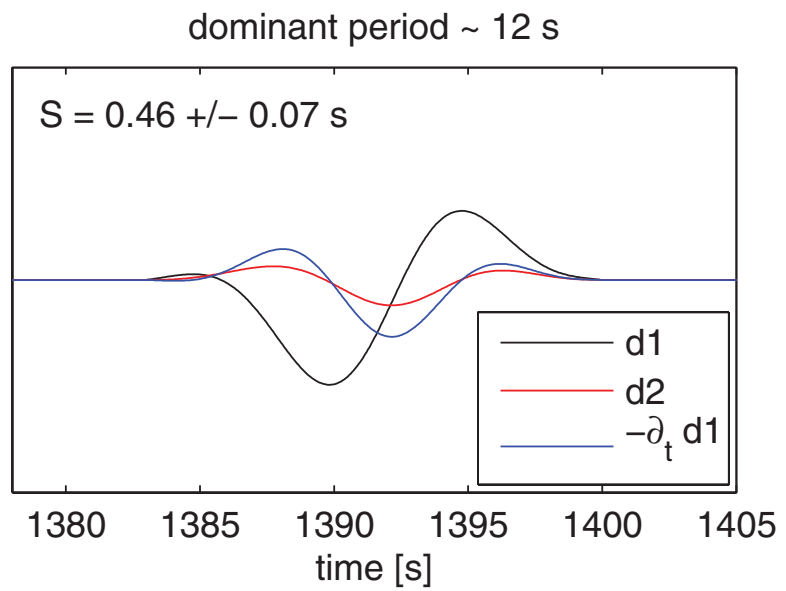

Figure 2. Measurement of the $S K S$-splitting intensity by projecting the transverse signal $\left(d_{2}\right)$ onto the time derivative of the radial signal $\left(\partial_{t} d_{1}\right)$. Note the similarity of these two waveforms in agreement with equation (6). The filtered, time-windowed $S K S$ signals are the same as in Figure 1. 
Chevrot's $(2000,2006)$ original definition. We introduce a minus sign to obtain a more direct comparison with the cross-correlation splitting intensity discussed in the next section. The splitting intensity is thus to first order related to the splitting parameters by

$$
S \simeq-\frac{1}{2} \Delta T \sin 2\left(\phi_{f}-\phi_{0}\right) .
$$

From this relation it is clear that the splitting intensity has the dimension of a time. The splitting intensity was originally introduced in a multichannel technique to retrieve the splitting parameters $\Delta T$ and $\phi_{f}$. By measuring $S$ for a wide range of $\phi_{0}$ and by fitting the different measurements with a sine curve, we can indeed obtain $\Delta T$ and $\phi_{f}$ (Chevrot, 2000). The splitting intensity may be measured at different periods. In Figure 2, we analyze the same seismograms as in Figure 1 using the projection method (equation 7). The uncertainties in the measurements are estimated from the difference between $d_{2}$ and $-S \dot{d}_{1}$ (Chevrot, 2000). The splitting intensities measured based upon this method (Fig. 2) can be compared to the values of $-\frac{1}{2} \Delta T \sin 2\left(\phi_{f}-\phi_{0}\right)$ as given by the twoparameter approach (Fig. 1) for weak anisotropy. There is agreement within the uncertainties of the measurements.

We will see in the section entitled Sensitivity that the Fréchet derivative of the splitting intensity with respect to the density and the elastic parameters may be obtained based upon the Born approximation (Dahlen et al., 2000; Favier and Chevrot, 2003; Tromp et al., 2005; Chevrot, 2006). Calculating the Fréchet derivatives relative to structural parameters is key to imaging schemes. Because we can calculate these derivatives (analytically or numerically), the $S K S$-splitting intensity has a great advantage over the twoparameter approach.

\section{The Cross-Correlation Splitting Intensity}

In Sieminski et al. (2007b), we proposed to use temporal observables, inspired by the cross-correlation travel-time anomaly, to analyze $S$-wave sensitivity to anisotropy. The observable we present here comes from the general definition for $S$ waves (equation 18 of Sieminski et al., 2007b). Before discussing this observable in the specific case of $S K S$ waves, we first review the definition and advantages of measuring travel-time anomalies by cross correlation.

From a practical perspective, to make a reliable crosscorrelation travel-time measurement, we need two similarly shaped waveforms. In global tomography, this may be accomplished by cross-correlating an observed long-period waveform with a corresponding synthetic reference waveform. The observed waveform $d$ recorded on a given component is expected to be very similar in shape to the reference waveform $s$ time shifted by an amount $\delta T$, such that we may write $d(t) \simeq s(t-\delta T)$. The travel-time anomaly $\delta T$ may be retrieved by cross-correlating the observed and reference signals. It corresponds to the maximum of the cross correlation,

$$
\Gamma(\tau)=\int s(t-\tau) d(t) \mathrm{d} t
$$

the integral being over the time window of the wave of interest. Cross correlation is generally preferred over picking for global body-wave tomography because it facilitates a precise and objective analysis of longer-period body waves that have a good signal-to-noise ratio (Woodward and Masters, 1991; Su and Dziewonski, 1992). It is a quick, easy, and very robust data-analyzing tool. The technique is derived from a matched filter optimally designed to detect a known wavelet in the presence of white noise (Robinson and Treitel, 1980). For meaningful results, the observed signal must be very similar to the reference signal. To assess this similarity, Maggi et al. (2006) proposed three indicators based upon the cross-correlation function $\Gamma$ (equation 9): (1) the maximum value $\max [\Gamma]$ of the cross-correlation function, (2) the value of $\delta T$ at which $\Gamma$ is maximum, and (3) the amplitude ratio $\delta \ln A$ between the observed and time-corrected reference signals. Acceptance limits for these indicators are then tuned depending on the problem and the dataset at hand, usually to mimic the selection that would be made by inspecting the data by eye. The uncertainties in the accepted measurements can be estimated from the difference between the observed signal $d(t)$ and the time-corrected reference signal $s(t-\delta T)$ through their variance,

$$
\sigma^{2}=\frac{\int[d(t)-s(t-\delta T)]^{2} \mathrm{~d} t}{\int \dot{s}^{2}(t-\delta T) \mathrm{d} t} .
$$

Together with carefully designed and tuned acceptance criteria, the cross-correlation method is well adapted for automated measurement algorithms (Ritsema and van Heijst, 2002; Maggi et al., 2006), making it possible to build large, reliable, and easily reproduced datasets. As the cross correlation involves finite time windows, the measured travel times are intrinsically finite-frequency data, unlike the infinitefrequency picked travel times. By cross-correlating bandpass-filtered signals in different period ranges, we also obtain frequency-dependent travel-time anomalies. Consistent analysis of these data therefore requires that we consider finite-frequency wave propagation. Dahlen et al. (2000) have shown how the cross-correlation travel-time anomaly may be related to the Earth's structure through finite-frequency sensitivity kernels. Our aim is to find a suitable observable that can be used to make robust finite-frequency $S K S$-splitting measurements and that may be related to the Earth's structure through complementary finite-frequency sensitivity kernels.

For weak anisotropy and to first order, equation (6) gives $d_{2} \simeq-S \dot{d}_{1}$, suggesting that there exists a time shift $\delta \tau$ such that

$$
d_{1}(t)+d_{2}(t) \simeq d_{1}(t)-\delta \tau \dot{d}_{1}(t) \simeq d_{1}(t-\delta \tau),
$$

and the splitting intensity $S$ can be measured by crosscorrelating the sum $d_{1}+d_{2}$ with $d_{1}$. Following Dahlen 
et al.'s (2000) Born formalism, in weakly anisotropic media we may interpret the transverse component $d_{2}$ as a (firstorder) scattered radial signal from $d_{1}\left(d_{2}=\delta s_{1} \simeq \delta d_{1}\right)$. Adding $d_{2}$ to $d_{1}$ slightly perturbs $d_{1}$, and in weakly anisotropic media, equation (11) shows that this perturbation is small enough to be interpreted as a time shift, which may be measured by cross correlation. We thus consider the crosscorrelation function

$$
\Gamma(\tau)=\int d_{1}(t-\tau)\left(d_{1}+d_{2}\right)(t) \mathrm{d} t
$$

Expecting a small $\delta \tau$, this cross correlation is calculated by integrating over the $S K S$ time window and it has only one maximum. Let us confirm that the time shift $\delta \tau$ of the maximum of the cross correlation (equation 12) is indeed the splitting intensity $S$ of equation (7) for weak anisotropy. The time shift $\delta \tau$ is defined by

$$
\partial_{\tau} \Gamma(\delta \tau)=-\int \dot{d}_{1}(t-\delta \tau)\left(d_{1}+d_{2}\right)(t) \mathrm{d} t=0
$$

dominant period $\sim 8 \mathrm{~s}$

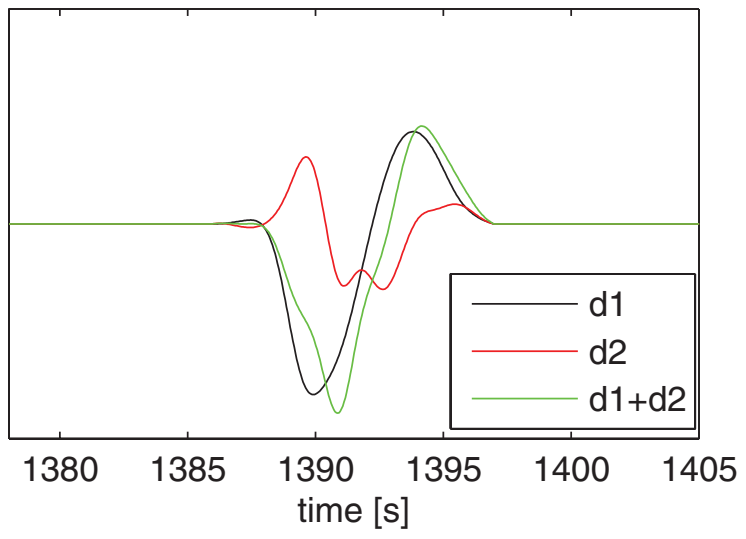

normalized cross-correlation function

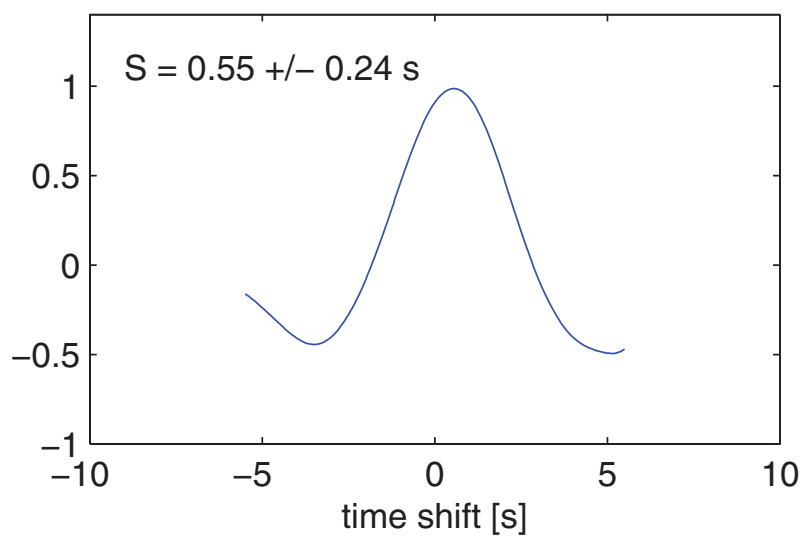

For weak anisotropy, this yields to first order

$$
\delta \tau \simeq-\frac{\int \dot{d}_{1}(t) d_{2}(t) \mathrm{d} t}{\int \dot{d}_{1}^{2}(t) \mathrm{d} t},
$$

which is the original definition of the splitting intensity (equation 7). Figure 3 shows an example of measuring the $S K S$-splitting intensity with the cross-correlation method for the seismograms shown in Figures 1 and 2. Note the similarity in the waveforms of the summed signal $d_{1}+d_{2}$ and the radial signal $d_{1}$ at these rather long periods, showing that it is sensible to cross-correlate these two signals. For the validity of the method, it is important to check this similarity. Acceptance of the measurements can be decided with Maggi et al.'s (2006) indicators adapted for the cross correlation in equation (12). For accepted measurements, uncertainties are then estimated with the variance $\sigma^{2}$ (equation 10) quantifying the similarity of $\left(d_{1}+d_{2}\right)(t)$ and the time-shifted $d_{1}(t-\delta \tau)$.

We have seen that for weak anisotropy, the $S K S$ splitting intensity can be measured from the cross correlation

$$
\text { dominant period } ~ 12 \mathrm{~s}
$$

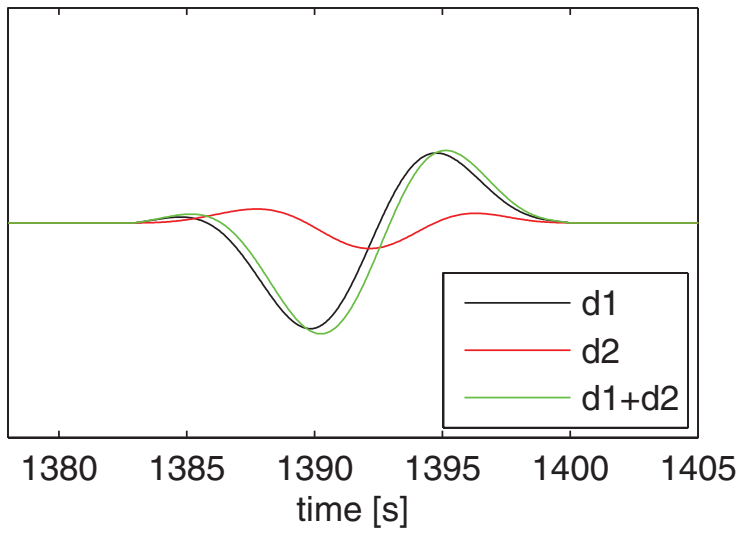

normalized cross-correlation function

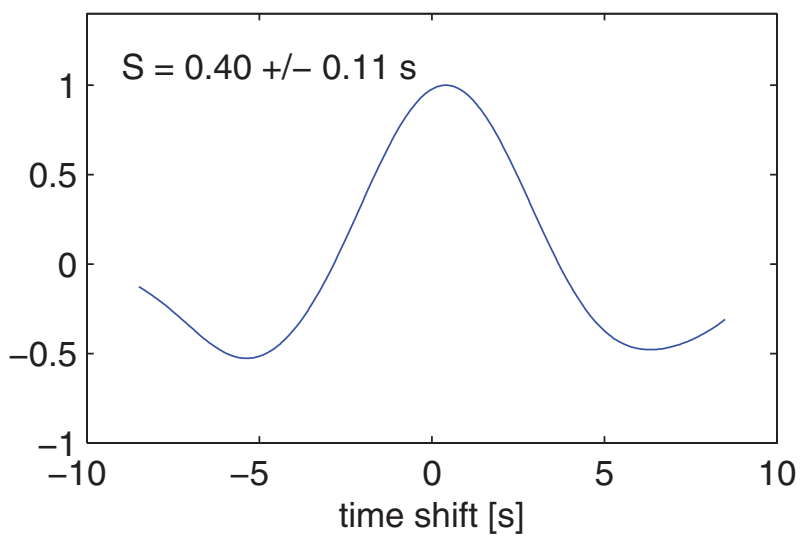

Figure 3. Measurement of the $S K S$-splitting intensity by cross-correlating the radial signal $\left(d_{1}\right)$ with the sum of the radial and transverse signal $\left(d_{1}+d_{2}\right)$. Note the similarity of these two waveforms, making it possible to cross-correlate them. The top panels show the filtered, time-windowed $S K S$ signals and the bottom panel the normalized cross-correlation function (equation 12), its length depending on the selected time window, which is different for these two periods. The signals are the same as in Figures 1 and 2. 
in equation (12). This method is validated based upon seismograms from the northern California dataset of Hartog and Schwarz (2000, 2001). We selected the recordings of 10 deep events (Table 1) at 13 of the BDSN stations (Fig. 4). Figure 5 compares the $S K S$-splitting intensities from the cross-correlation method to the measurements from the two previous methods in different period ranges (from periods longer than $1 \mathrm{sec}$ to periods longer than $13 \mathrm{sec}$ ). The cross-correlation measurements are accepted if they satisfy the following criteria: (1) $\max [\Gamma]$ must be larger than $0.95,(2)|\delta \tau|$ must be smaller than $2 \mathrm{sec}$, and (3) $|\delta \ln A|$ must smaller than 0.5 . These values have been chosen from visual inspection of the waveforms (indicator 1), the previously observed values of splitting intensity (Chevrot, 2000; Long and van der Hilst, 2005) (indicator 2), and the assumption $d_{2} \ll$ $d_{1}$ (indicator 3). For this dataset, the measurements are mostly rejected because $\max [\Gamma]$ is too small. For periods longer than $1 \mathrm{sec}$ (signals band-pass filtered between 1 and $100 \mathrm{sec}$ ), fewer cross-correlation measurements are accepted, because at short period the waveforms are indeed more complicated and less similar (Fig. 3). The larger uncertainties obtained for periods longer than $13 \mathrm{sec}$ (signals band-pass filtered between 13 and $100 \mathrm{sec}$ ) result from the small amplitude of the $S K S$ signal in this period range for these events. For the two-parameter measurements, we use the energy-minimization technique illustrated in Figure 1. Only the most reliable measurements are kept. They are identified from the $95 \%$ confidence values of $\phi_{f}$ and $\Delta T$ obtained based upon an F-test. We discard measurements with an error larger than $45^{\circ}$ in the fast polarization or an error larger than $2 \mathrm{sec}$ in the time shift. It is usually the error in the fast polarization that is too large for this dataset. This technique selects fewer measurements, especially at longer periods where the signal amplitude is small (Fig. 1). This is another advantage of the splitting intensity over the traditional twoparameter description, because it gives acceptable results $(S \simeq 0)$ close to null measurements. Within the uncertainties, the cross-correlation measurements are in good agreement

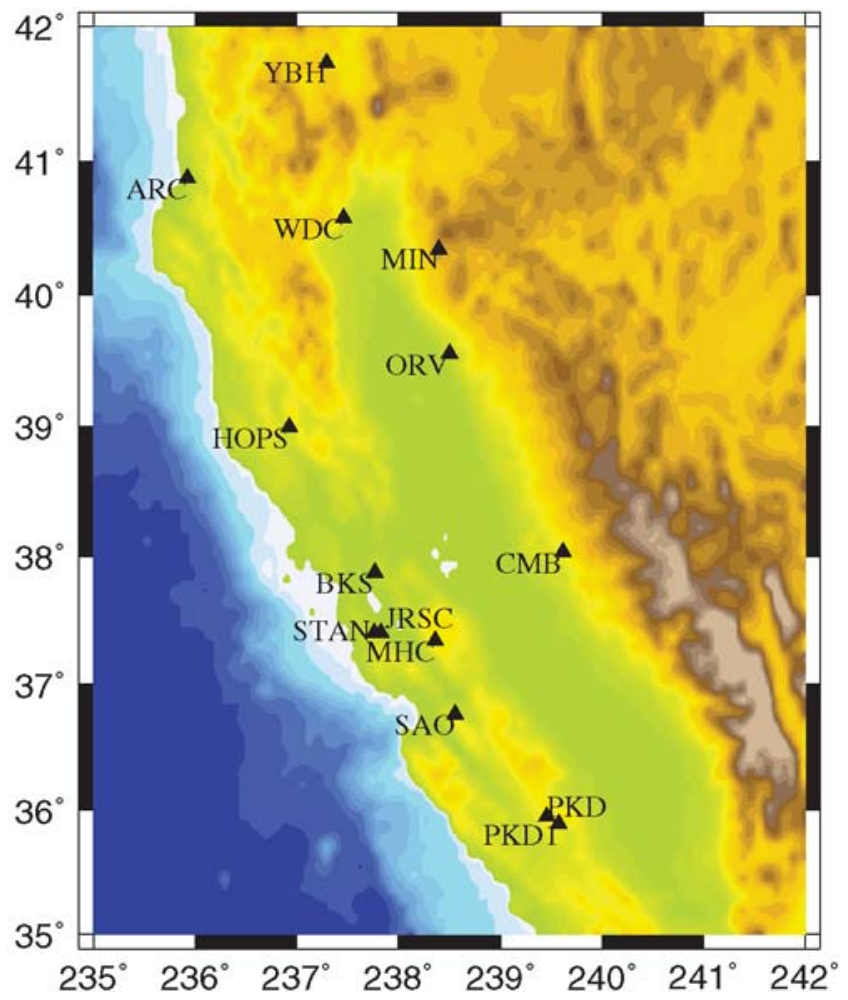

Figure 4. Map of the 13 BDSN stations used to obtain the measurements shown in Figure 5.

with the results from the other two methods (Fig. 5). The agreement is especially good for dominant periods between 1 and $13 \mathrm{sec}$. For large splitting intensities $(|S|>1 \mathrm{sec})$, the agreement slightly deteriorates, as is clearly seen for periods longer than $13 \mathrm{sec}$. This is not surprising, because the different definitions of the splitting intensities (equations 7, 8, and 12) are equivalent under the assumption of weak anisotropy (and first-order calculations). For dominant periods between 1 and $13 \mathrm{sec}$ the splitting intensities of real data are usually small and satisfy the weak-anisotropy condition.

Table 1

Ten Earthquakes Selected from the Hartog and Schwarz $(2000,2001)$ Dataset for the Measurements Shown in Figure 5

\begin{tabular}{llrrrrrc}
\hline Event Name & Region & Latitude $\left(^{\circ}\right)$ & Longitude $\left({ }^{\circ}\right)$ & Depth $(\mathrm{km})$ & $M_{b}$ & Distance $\left(^{\circ}\right)$ & Back Azimuth $\left(^{\circ}\right)$ \\
\hline 080793A & Taiwan & 26.68 & 125.84 & 164.90 & 6.0 & 89.4 & 304.1 \\
080993G & Hindu Kush & 36.38 & 70.47 & 213.70 & 5.8 & 104.5 & 349.7 \\
090693A & New Ireland & -4.78 & 153.13 & 59.20 & 6.0 & 89.0 & 263.1 \\
062495A & New Ireland & -3.83 & 153.93 & 386.90 & 6.2 & 87.8 & 263.4 \\
062995D & Vanuatu & -19.42 & 168.95 & 142.70 & 6.2 & 86.6 & 242.1 \\
081495A & New Britain & -4.90 & 151.80 & 140.40 & 6.3 & 90.1 & 263.9 \\
110596B & Kermadec & -30.95 & -179.73 & 366.70 & 5.9 & 87.6 & 226.7 \\
050397C & Kermadec & -31.70 & -179.06 & 119.30 & 6.6 & 87.8 & 225.8 \\
052597D & Kermadec & -32.02 & -179.95 & 345.00 & 6.2 & 88.5 & 226.1 \\
040599A & New Britain & -5.65 & 149.71 & 149.40 & 6.2 & 92.2 & 264.6 \\
\hline
\end{tabular}

Most events are located in the southwest Pacific subduction zone. The event parameters are taken from the Centroid Moment Tensor catalog (www.globalcmt.org). The average epicentral distance and back azimuth for the 13 BSDN stations shown in Figure 4 are indicated. 

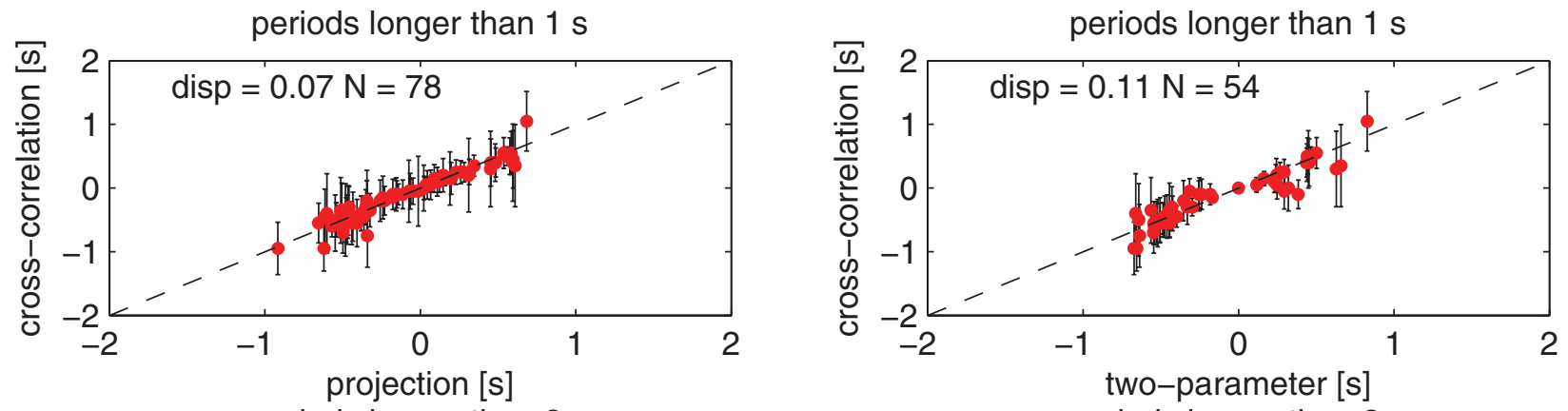

periods longer than $3 \mathrm{~s}$
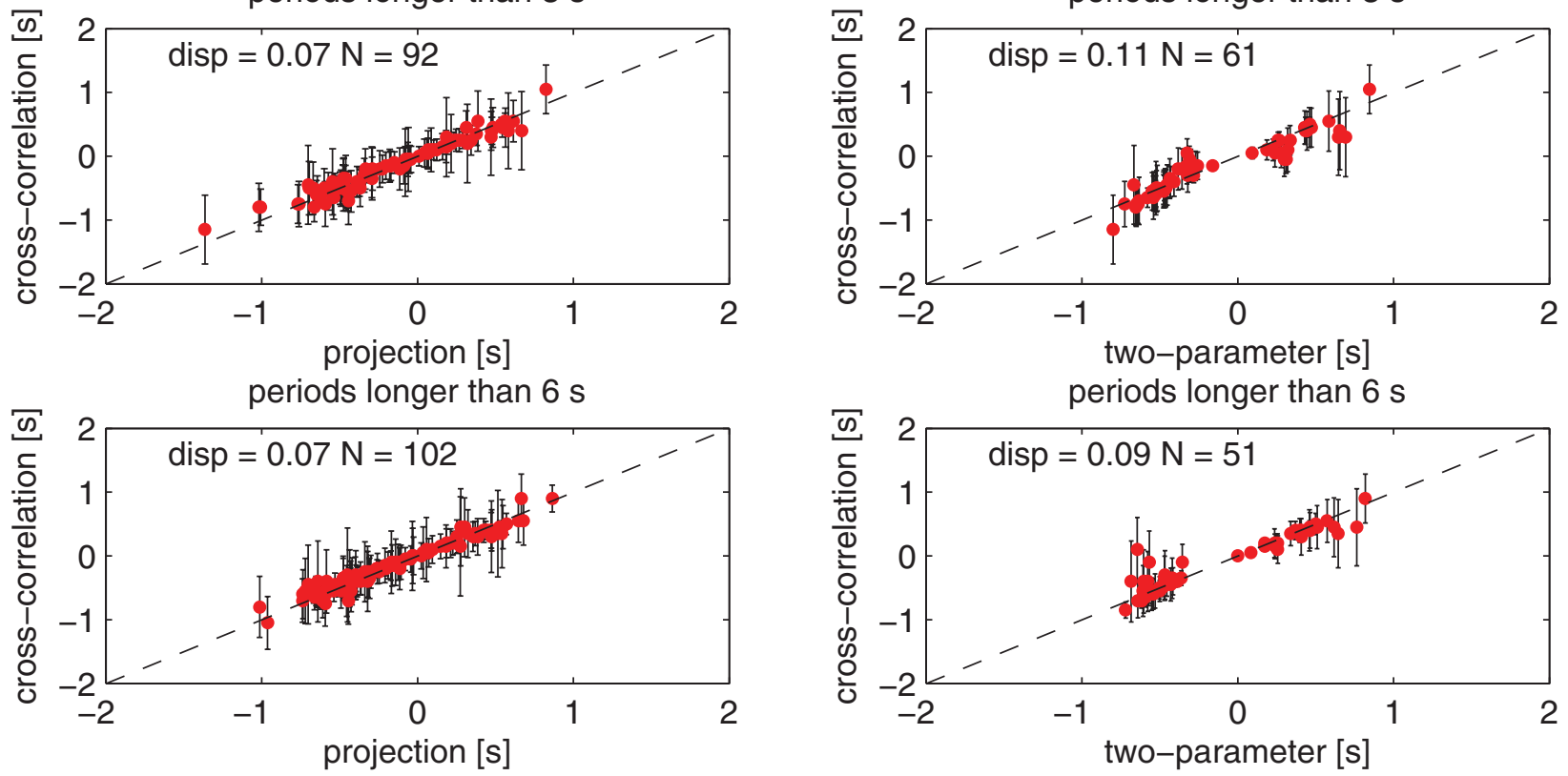

periods longer than $6 \mathrm{~s}$
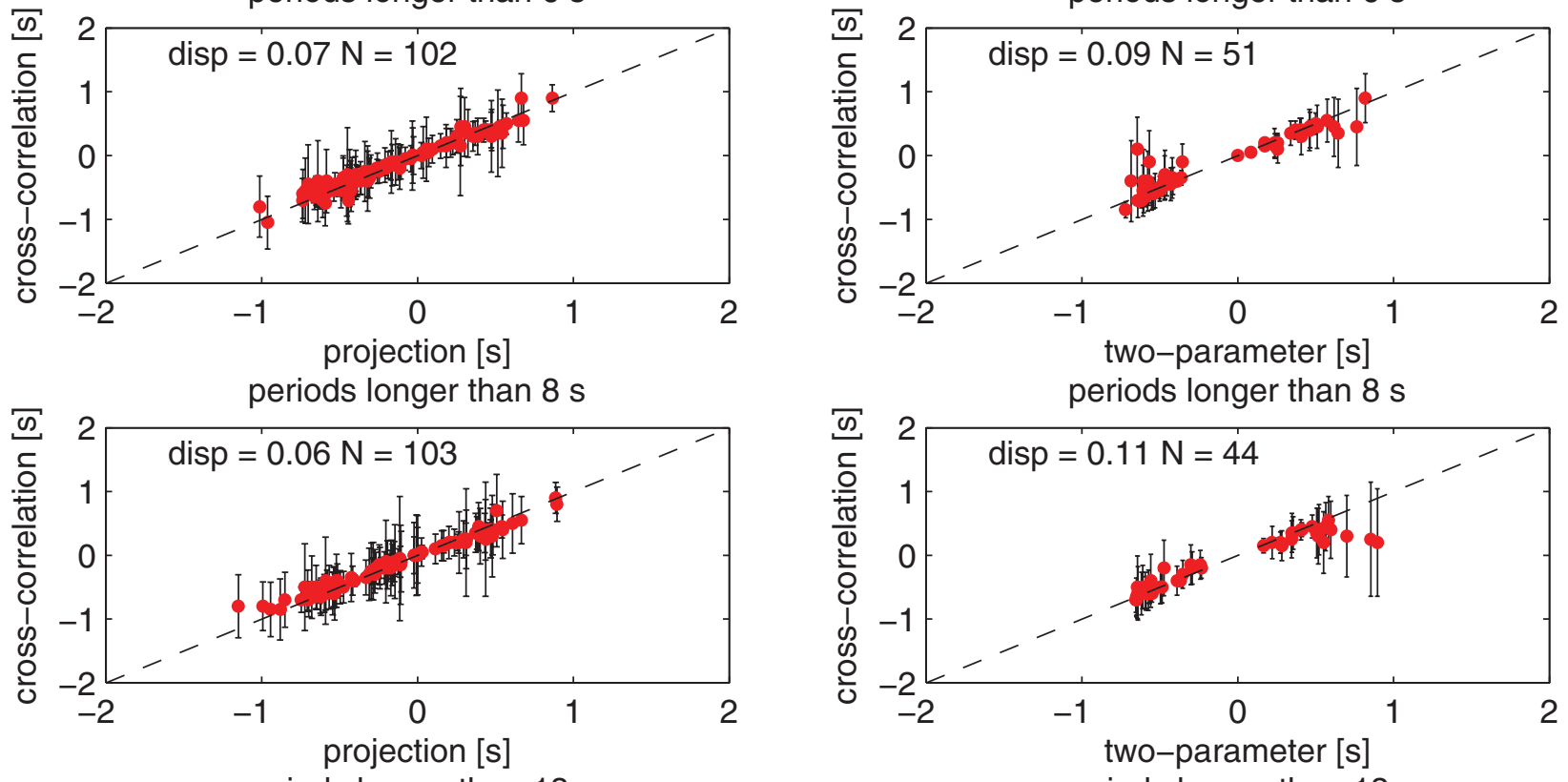

periods longer than $13 \mathrm{~s}$
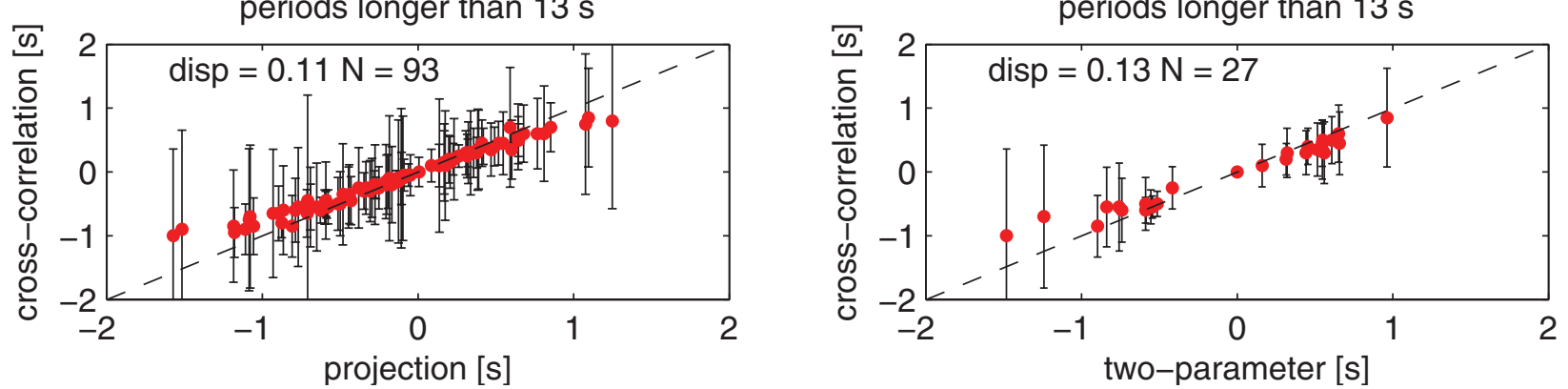

Figure 5. Comparison of the $S K S$-splitting intensities measured based upon the cross-correlation method and the projection method (lefthand side) and the two-parameter approach (right-hand side) at different periods. The error bars are calculated from equation (10) adapted for the cross-correlation measurements (i.e., $d=d_{1}+d_{2}$ and $s=d_{1}$ ). The number $N$ is the number of accepted measurements and disp is the mean difference between the measurements with the cross-correlation method and the results of the two others' methods. The data are from the northern California dataset of Hartog and Schwarz (2000, 2001). Ten deep events (Table 1) have been analyzed at 13 of the BDSN stations (Fig. 4). 
This experiment with real data validates cross correlation as a suitable technique to measure the $S K S$-splitting intensity. This way of measuring the $S K S$-splitting intensity fully benefits from the advantages of the cross-correlation method described before, that is, the finite-frequency content, robustness, and the possibility to automate the procedure to obtain large datasets. All of this can also be said for the projection method (equation 7), but a practical advantage of measuring the splitting intensity by cross correlation is that it is a straightforward adaptation of the cross-correlation technique widely used to measure travel-time anomalies for which many tools have already been designed. We selected the $S K S$ time window by visual inspection for the experiment shown in Figure 5. But this step could also have been automated based upon Maggi et al.'s (2006) windowselection algorithm. Their algorithm uses the short-term/ long-term ratio of the signal envelope to detect windows containing arrivals. This can easily be applied to the $S K S$ signal recorded on the radial component. In the next section, we investigate the suitability of the $S K S$-splitting intensity for imaging mantle anisotropy.

\section{Sensitivity}

\section{The Adjoint Spectral-Element Method}

For first-order perturbations, the sensitivity of the $S K S$ splitting intensity is given by sensitivity kernels $K$ describing how perturbations in structural parameters $m$ affect the observable

$$
\delta S=\int K(\mathbf{x}) \delta m(\mathbf{x}) \mathrm{d}^{3} \mathbf{x},
$$

where $\mathbf{x}$ is the spatial location and the integral is over the Earth's volume. The sensitivity kernel is the Fréchet derivative of the observable and $K=\partial_{m} S$. Replacing $d_{1}$ by $s_{1}+$ $\delta s_{1}$ (equation 4) and $d_{2}$ by $\delta s_{2}$ (equation 5) in equation (14) or equation (7), and keeping first-order terms only, we may write

$$
\partial_{m} S \simeq-\frac{\int \dot{s}_{1}(t) \partial_{m} s_{2}(t) \mathrm{d} t}{\int \dot{s}_{1}^{2}(t) \mathrm{d} t} .
$$

Favier and Chevrot (2003) have developed an analytical expression for the sensitivity kernels of the $S K S$-splitting intensity to some anisotropic parameters. They determined the Fréchet derivative of the transverse signal $\partial_{m} s_{2}$ in equation (16) with respect to the elastic parameters using the Born approximation and a plane-wave description. Their analysis is limited to hexagonal symmetry with a horizontal axis, and more specifically to two elastic parameters, $\gamma_{c}$ and $\gamma_{s}$. These parameters are related to Thomsen's (1986) parameter $\gamma$, representing $S$-wave anisotropy in media with hexagonal symmetry, by $\gamma_{c}=\gamma \cos 2 \xi_{0}$ and $\gamma_{s}=\gamma \sin 2 \xi_{0}$, where $\xi_{0}$ is the azimuth of the symmetry axis.
We adopt here a more general approach. We numerically compute the sensitivity kernels to 21 elastic parameters considering general anisotropy. The computation is based upon the combination of adjoint methods with spectral-element simulations of wave propagation (Komatitsch and Vilotte, 1998; Komatitsch and Tromp, 1999). Details of this method are given in Tromp et al. (2005) and Liu and Tromp (2006). In this approach, the sensitivity kernels—-the Fréchet derivatives-for a seismic observable at a given receiver are constructed from the interaction of the regular wave field, propagating from the source to the receiver, with the adjoint wave field, propagating from the receiver to the source. The propagation of the wave field is simulated with a spectralelement method. The expression for the interaction of the two wave fields depends on the structural parameters, and the adjoint wave field is generated by a point-force source depending on the observation. For an observable $o$ whose Fréchet derivative is

$$
\partial_{m} o=\int h(t) \partial_{m} s_{i}(t) \mathrm{d} t
$$

where $s_{i}$ is the reference signal on component $i$ and $h(t)$ a function depending on the observable, the adjoint source time function $\mathbf{f}^{\dagger}$ is the function $h$ reversed in time (Luo and Schuster, 1991; Tromp et al., 2005)

$$
\mathbf{f}^{\dagger}(t)=h(T-t) \hat{\mathbf{e}}_{i},
$$

with $T$ the duration time of interest and $\hat{\mathbf{e}}_{i}$ the unit vector corresponding to component $i$. Following equations (17) and (18), the adjoint source time function for the $S K S$-splitting intensity with the Fréchet derivative of equation (16) is

$$
\mathbf{f}^{\dagger}(t)=-\frac{\dot{s}_{1}(T-t)}{\int \dot{s}_{1}^{2}(t) \mathrm{d} t} \hat{\mathbf{e}}_{2} .
$$

The sensitivity kernels for the $S K S$-splitting intensity are therefore calculated by sending back the time derivative of the reference radial signal at the receiver applied on the transverse direction.

To calculate the sensitivity kernels, we must also choose how to parameterize the elastic structure. We describe anisotropy with the 21 elastic parameters naturally involved in asymptotic wave propagation in weakly anisotropic media (Table 2). Each of these parameters is given by an independent combination of the components of the elastic tensor (Chen and Tromp, 2007). This parameterization of anisotropy highlights the directional dependence of asymptotic propagation in anisotropic media. In the simplest anisotropic model, that is, hexagonal symmetry, asymptotic propagation depends on the incidence angle relative to the symmetry axis. For example, when the symmetry axis is vertical (or radial), $S H$ waves propagate horizontally with a speed given by $\sqrt{N / \rho}$ (with $\rho$ the density), while the speed is $\sqrt{L / \rho}$ for $S$ waves propagating vertically. In more general anisotropic 
Table 2

The 21 Elastic Parameters Describing Asymptotic Propagation in Weakly Anisotropic Media (Chen and Tromp, 2007) and Their Associated Azimuthal Dependence

\begin{tabular}{lc}
\hline \multicolumn{1}{c}{ Elastic Parameters } & Azimuthal Dependence \\
\hline$A C N L F$ & $0 \xi$ \\
$J_{c} J_{s} K_{c} K_{s} M_{c} M_{s}$ & $1 \xi$ \\
$B_{c} B_{s} H_{c} H_{s} G_{c} G_{s}$ & $2 \xi$ \\
$D_{c} D_{s}$ & $3 \xi$ \\
$E_{c} E_{s}$ & $4 \xi$ \\
\hline
\end{tabular}

The angle $\xi$ is the azimuth along the geometrical ray path. Each of these parameters is a linear combination of the components of the elastic tensor (Chen and Tromp, 2007). All of them involve a directional dependence of the wave speed. The parameters $A, C, N, L$, and $F$ fully describe anisotropy with hexagonal symmetry and a vertical (or radial) symmetry axis. They do not cause any azimuthal dependence. The parameters producing azimuthal anisotropy are organized in pairs: the $c$ parameters correspond to a dependence in $\cos n \xi$, and the $s$ parameters correspond to a dependence in $\sin n \xi$ (with $n=1,2$, 3 , and 4).

models, asymptotic propagation also depends on the azimuth. This azimuthal dependence has been widely studied for surface waves (Smith and Dahlen, 1973; Montagner and Nataf, 1986). Analyses of surface-wave (Sieminski et al., 2007a) and body-wave sensitivity (Sieminski et al., 2007b) to anisotropy show that finite-frequency propagation presents the same type of directional dependence. Seismic travel times are more efficiently described by wave speed rather than elastic parameters and density (Tromp et al., 2005; Liu and Tromp, 2006). Because the splitting intensity may be seen as a temporal observable, we prefer to consider the squared wave speeds associated with the asymptotic parameters of Table 2. These 21 anisotropic squared wave speeds are simply the density-normalized elastic parameters. We denote them with a prime, for example, $A^{\prime}=A / \rho$.

The adjoint spectral-element method makes it possible to compute sensitivity kernels in any 3D anisotropic model. The sensitivity pattern may be strongly affected by 3D structures (Zhao et al., 2005; de Hoop et al., 2006; Liu and Tromp, 2006). To identify the fundamental characteristics of the sensitivity of the $S K S$-splitting intensity, it is, however, more appropriate to analyze the kernels in a simple Earth model, and we choose the isotropic preliminary reference Earth model (PREM) (Dziewonski and Anderson, 1981).

\section{Favorable Properties for Imaging}

Body-wave travel-time sensitivity to isotropic perturbations in 1D reference models has been described by a banana-doughnut kernel (Marquering et al., 1999), that is, body-wave travel times are sensitive to the structure over a volume around the geometrical ray path with the sensitivity being maximum off of the ray path and zero along the ray path (Hung et al., 2000; Liu and Tromp 2006; Zhao and Jordan 2006). The doughnut pattern, however, disappears in the vicinity of the source and receiver because of near-field effects (Favier et al., 2004). The width of the banana and of the doughnut hole depend on the path length and the wave period. For anisotropy, the general sensitivity pattern is still a banana doughnut in 1D reference models, but it is strongly controlled by a directional dependence (Sieminski et al., 2007b). The amplitude of the sensitivity asymptotically varies with the incidence angle and azimuth of the propagation. The sensitivity kernels for the $S K S$-splitting intensity exhibit these general properties, but the specific path geometry of $S K S$ waves lends additional characteristics to this observable.

First, the $S K S$-splitting intensity can only be sensitive to structural perturbations located between the CMB and the surface, because as the $S K S$ wave enters the liquid outer core, it loses its transverse component. This is confirmed by computation, the $S K S$-splitting kernels are a portion of a banana (Sieminski et al., 2007b), and we can focus on the receiver region (Fig. 6). The $S K S$ propagation is nearly vertical beneath the receiver. For common epicentral distances, the incidence angle is smaller than $11^{\circ}$ at the surface. For such incidence angles, the splitting intensity has significant sensitivity to only four pairs of parameters: $K_{c, s}^{\prime}, M_{c, s}^{\prime}, G_{c, s}^{\prime}$, and $D_{c, s}^{\prime}$ (Fig. 6). This is quite remarkable when compared to direct $P$ and $S$ waves that are sensitive to far more parameters (Sieminski et al., 2007b) and surface waves (Sieminski et al., 2007a). In Figure 6 we only display the sensitivities to the $s$ parameters. The sensitivities to the $c$ parameters, while being different, have the same characteristics. Differences will be discussed in Figure 7.

The high sensitivity to these eight parameters close to the receiver (Fig. 6) is due to the combined effects of geometrical focusing, directional dependence, and near-field terms. The area of significant sensitivity has a limited extent in depth. For the $105^{\circ}$ path shown in Figure 6 (also shown in Figure 7), the high-sensitivity zone (the dark red or dark blue regions) reaches $550 \mathrm{~km}$ with a width of about $380 \mathrm{~km}$ for the parameter $G_{s}^{\prime}$ for periods longer than $8 \mathrm{sec}$. The depth extent of significant sensitivity is, however, controlled by the directional dependence and wave period (Fig. 7). For example, the azimuthal dependence of the sensitivity to $G_{s}^{\prime}$ and $G_{c}^{\prime}$ is asymptotically given by $\cos 2 \xi$ and $\sin 2 \xi$, respectively (with $\xi$ the azimuth along the geometrical ray path). When the azimuth $\xi_{r}$ in the vicinity of the receiver is close to $180^{\circ}$, as for the path in Figure 6, the azimuthal variation of the depth extent reaches a maximum for $G_{s}^{\prime}$ and a minimum for $G_{c}^{\prime}$ (Fig. 7). Note that for a path with $\xi_{r}$ close to $90^{\circ}$ the depth extent of the sensitivity to $G_{s}^{\prime}$ is also maximum but the sensitivity amplitude has the opposite sign (Fig. 7). For the parameters $G_{c, s}^{\prime}$, the more vertical the path (i.e., the larger the epicentral distance), the higher the sensitivity. But this effect is small over the typical distance range because, for paths between $85^{\circ}$ and $115^{\circ}$, the incidence does not vary much (from $6^{\circ}-11^{\circ}$ at the surface). At longer periods (Fig. 7), the significant sensitivity zone is wider, but it is still limited to upper-mantle depths. We especially note that although the 
$\mathrm{A}^{\prime}$

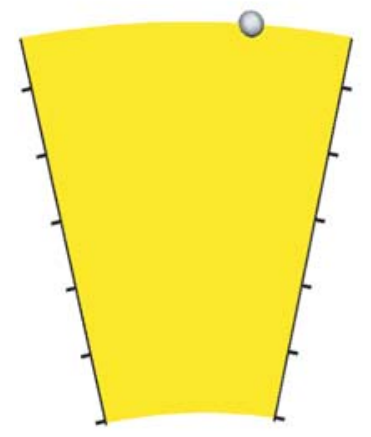

$F^{\prime}$

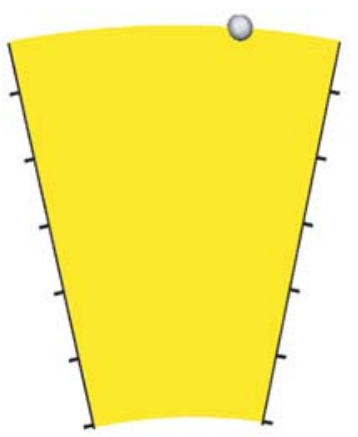

Bs'

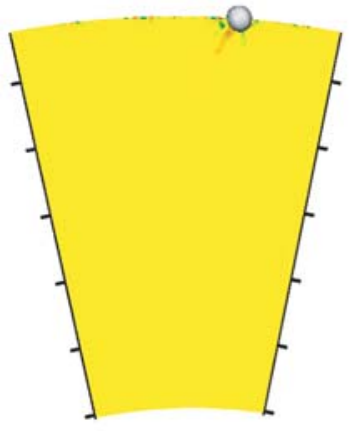

Es'

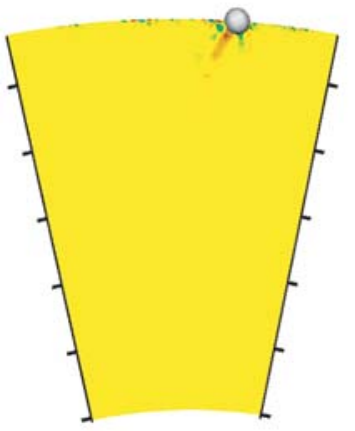

$\mathrm{C}^{\prime}$

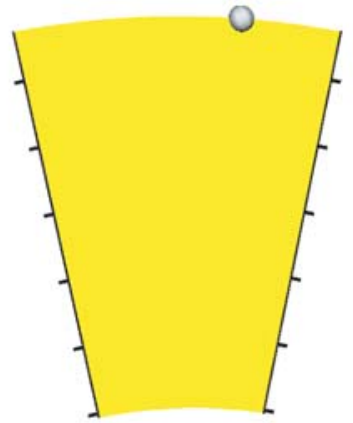

Js'

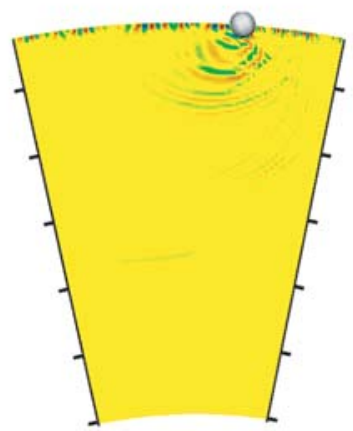

Hs'

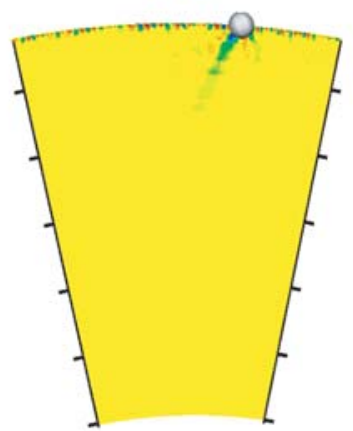

$\mathrm{N}^{\prime}$

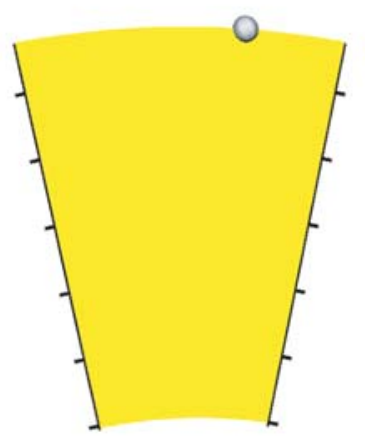

$\mathrm{Ks}^{\prime}$

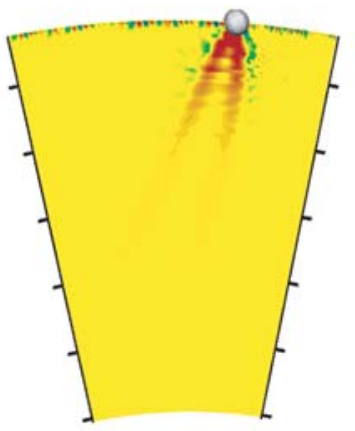

Gs'

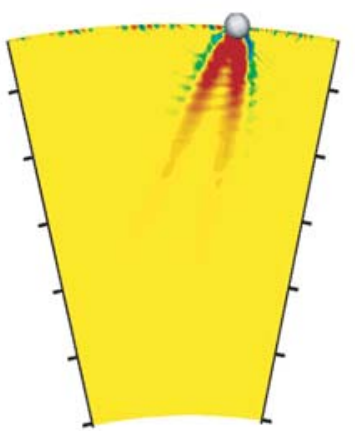

$\mathrm{L}^{\prime}$

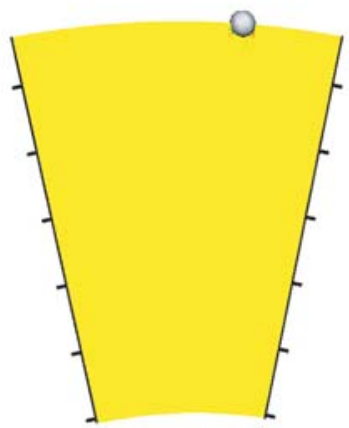

Ms'

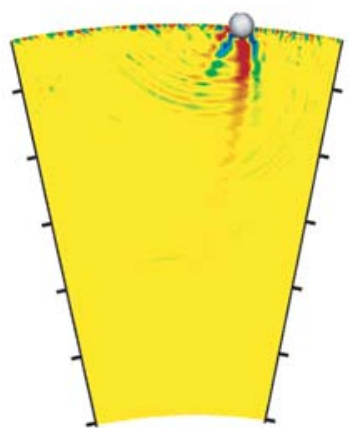

Ds'

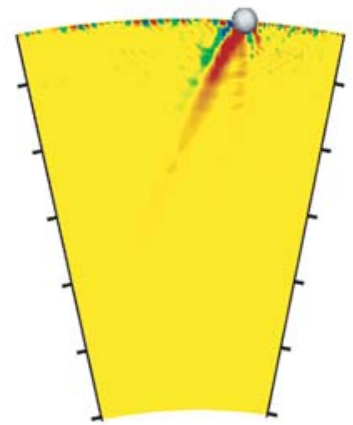

sensitivity $\left(\mathrm{s}^{3} \mathrm{~km}^{-5}\right)$

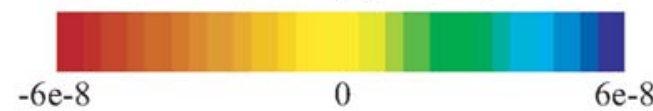

Figure 6. $S K S$-splitting intensity sensitivity kernels for 13 of the asymptotic squared wave speeds (Table 2). Only the $s$ parameters for azimuthal anisotropy are shown. The kernels are displayed on a depth section from the CMB to the surface along the source-receiver great circle path. We zoom in on the receiver region. The depth tick marks are situated every $500 \mathrm{~km}$ from the CMB to the surface, that is, the first and second tick marks from the surface are at 400 - and $900-\mathrm{km}$ depth, respectively. The epicentral distance is $105^{\circ}$ and the azimuth $\xi_{r}$ at the receiver (the small gray sphere) is $170^{\circ}$. The source mechanism is the 9 June 1994 Bolivia earthquake located at 647-km depth. The adjoint spectral-element computation is performed for periods longer than $8 \mathrm{sec}$. The sensitivity is significant only for $G_{s}^{\prime}, K_{s}^{\prime}, M_{s}^{\prime}$, and $D_{s}^{\prime}$ (and $G_{c}^{\prime}$, $K_{c}^{\prime}, M_{c}^{\prime}$, and $D_{c}^{\prime}$. 

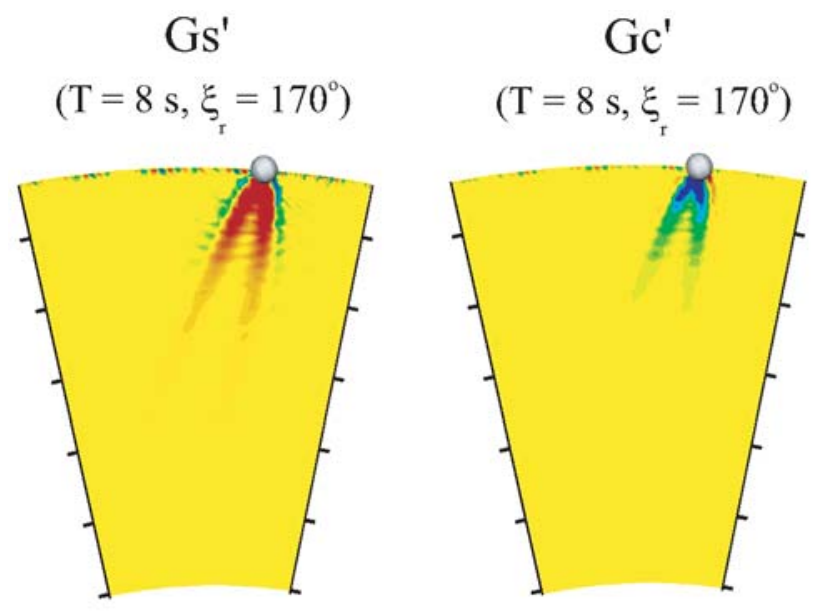
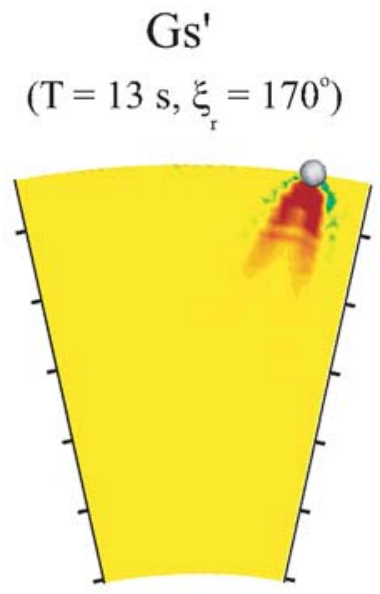

$$
\left(\mathrm{T}=13 \mathrm{~s}, \xi_{\mathrm{r}}=99^{\circ}\right)
$$

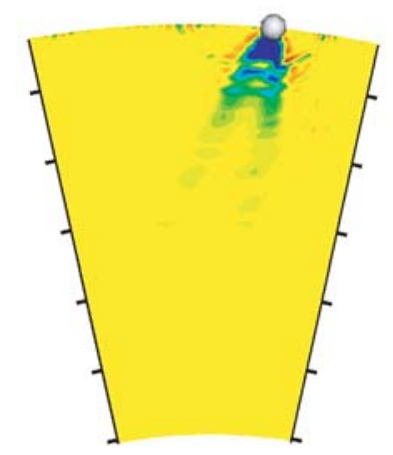

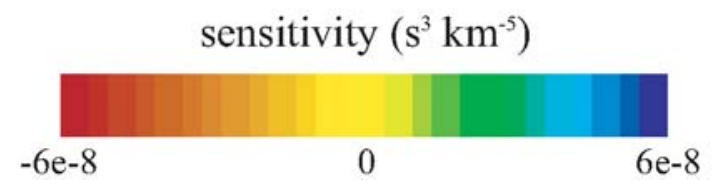

Figure 7. Variation with azimuth and period of the $S K S$-splitting sensitivity to the parameters $G_{s, c}^{\prime}$. The period $T$ is the minimum period of the adjoint spectral-element computation, and $\xi_{r}$ denotes the azimuth at the receiver. The sensitivity to $G_{s}^{\prime}$ and $G_{c}^{\prime}$ varies asymptotically with $\cos 2 \xi$ and $\sin 2 \xi$, respectively. The first image from left is the same as in Figure 6 . The zone of significant sensitivity (dark red or dark blue regions) is limited to the transition zone (about 550-km depth in the first image, $300 \mathrm{~km}$ in the second image, and $400-\mathrm{km}$ depth in the last two images).

sensitivity is not strictly zero in the $D^{\prime \prime}$ layer, it is nonsignificant compared to the sensitivity in the upper mantle. Single-receiver $S K S$-splitting measurements alone are thus unlikely to constrain the $D^{\prime \prime}$ region well.

Adjoint sensitivity kernels may exhibit numerous structures off the first Fresnel zone. Some of these are due to structural discontinuities in the reference model (isotropic PREM), for example, the oscillating positive and negative small-scale structures near the surface (Figs. 6 and 7). These structures are organized as rings around the receiver and are confined to the crust. They are due to scattered waves trapped in the crust, which arrive at the receiver in the selected $S K S$ time window. Because the adjoint method is a full-wave approach, it naturally considers the sensitivity of all of the waves that arrive in the selected window. Accordingly, these crustal sensitivity structures do not show up if the kernels are computed in a model without Moho discontinuity. This effect is more important at $8 \mathrm{sec}$ than at $13 \mathrm{sec}$ (Fig. 7) because the shorter period wave field is more affected by the presence of the crust. This means that short-period $(<10 \mathrm{sec}) S K S$ splitting data are sensitive to small-scale crustal heterogeneities. The circular patterns visible at different depths in the mantle in the depth sections shown in Figures 6 and 7 are also observed in a smooth mantle model (without wave speed discontinuities nor gradients). This suggests that these patterns are likely caused by reflected waves at the free surface from incident SKP waves that are backscattered in the mantle and reach the receiver in the $S K S$ time window (Sieminski et al., 2007b).
In anisotropic media with hexagonal symmetry and a horizontal axis, the parameters $K_{c, s}^{\prime}, M_{c, s}^{\prime}$, and $D_{c, s}^{\prime}$ are zero. In this case, the only relevant parameters for the $S K S$ splitting intensity are thus $G_{c, s}^{\prime}$, in agreement with previous work (Montagner et al., 2000; Favier and Chevrot, 2003). For this very specific type of anisotropy, the parameters $G_{c, s}^{\prime}$ are directly linked to Favier and Chevrot's (2003) parameters $\gamma_{c, s}$, such that $G_{c}^{\prime}=-\beta^{2} \gamma_{c}$ and $G_{s}^{\prime}=-\beta^{2} \gamma_{s}$ (with $\beta$ the isotropic $S$-wave speed). The sensitivity kernels for the two parameterizations are also simply related by

$$
\begin{aligned}
& K_{\gamma_{c}}=\beta^{2}\left(2 K_{H_{c}^{\prime}}-K_{G_{c}^{\prime}}\right) \simeq-\beta^{2} K_{G_{c}^{\prime}}, \\
& K_{\gamma_{s}}=-\beta^{2}\left(2 K_{H_{s}^{\prime}}-K_{G_{s}^{\prime}}\right) \simeq \beta^{2} K_{G_{s}^{\prime}},
\end{aligned}
$$

because $K_{H} \simeq 0$ for the $S K S$-splitting intensity (Fig. 6). With these relations, our numerical results can be readily compared to those of Favier and Chevrot (2003). There are differences, however. The numerical computation takes fully into account the directional dependence and all other complexities of wave propagation, such as free-surface and nearfield effects. Favier and Chevrot (2003) do not consider these effects. Their description captures the major part of the azimuthal dependence of the sensitivity, but it misses the part of the dependence with incidence. Because they consider plane waves, the incidence angle of the incoming wave is fixed. The variation of the incidence along the path beneath the receiver in the upper mantle is indeed small (for a $105^{\circ}$ path it varies from $20^{\circ}$ at $1000-\mathrm{km}$ depth to $8^{\circ}$ at the surface), but it 
nevertheless partly controls the depth extent of the sensitivity zone.

\section{Conclusions}

We proposed an alternative technique to measure the splitting intensity of $S K S$ waves. This observable quantifies the perturbation of the transverse signal. Although it cannot be directly interpreted in terms of the anisotropic properties, the $S K S$-splitting intensity is linearly linked to first-order structural perturbations, which makes it appropriate for imaging. We showed that the splitting intensity corresponds to a temporal observable that can be measured by crosscorrelating the radial signal with the sum of the radial and transverse signals. The cross-correlation measurements are in good agreement with the more traditional measurements for a northern California dataset. Like the cross-correlation travel-time anomaly, the cross-correlation $S K S$-splitting intensity is a simple, fast, and robust measure in the presence of noise. With an adapted window-selection algorithm and well-tuned acceptance criteria, it can be automated, making it possible to build large and reliable $S K S$-splitting datasets. By measuring this intrinsically finite-frequency observable at different periods, we fully benefit from the frequencydependent properties of wave propagation for imaging anisotropy. In addition, the $S K S$-splitting intensity presents favorable properties for imaging, as highlighted by a numerical computation of the sensitivity to general anisotropy. The significant sensitivity is focused on a small region limited to the upper mantle and on a small number of elastic parameters. Only two parameters are needed for anisotropy with hexagonal symmetry and a horizontal axis.

The sensitivity to anisotropy is strongly controlled by a directional dependence. It is difficult to assess whether all of the different directional effects are fundamental for imaging. Approximate analytical expressions, such as those in Favier and Chevrot (2003) and Chevrot (2006), may be sufficient to describe $S K S$-splitting sensitivity in isotropic 1D reference models. On the other hand, an iterative inversion scheme is needed for high-resolution imaging (de Hoop et al., 2006; Chen et al., 2007; Tape et al., 2007). Such an inversion scheme accounts for the nonlinearity of the tomographic problem, but for good convergence it requires the computation of the Fréchet derivatives - the sensitivity kernels-in 3D structures. This is quite challenging for analytical descriptions. Numerical methods, however, naturally consider the full physics of 3D wave propagation and accurately model the complexity of the sensitivity to anisotropy. They should thus constitute the basis for future high-resolution imaging of anisotropy.

\section{Acknowledgments}

We thank Guust Nolet and an anonymous reviewer for helpful comments and suggestions. The BDSN waveform data have been obtained thanks to the Northern California Earthquake Data Center (NCEDC). The adjoint spectral-element computations discussed in this article were per- formed on Caltech's Division of Geological \& Planetary Sciences Dell cluster. The source code for the adjoint spectral-element simulations is freely available from www.geodynamics.org. We gratefully acknowledge support from the European Commission's Human Resources and Mobility Programme, Marie Curie Research Training Networks, FP6, and from the National Science Foundation under Grant Number EAR-0711177. This is contribution Number 9002 of the Division of Geological \& Planetary Sciences, California Institute of Technology.

\section{References}

Alsina, D., and R. Snieder (1995). Small-scale sublithospheric continental mantle deformation: constraints from $S K S$ splitting observations, Geophys. J. Int. 123, 431-448.

Babuška, V., and M. Cara (1991). Seismic Anisotropy in the Earth, Kluwer Academic, Dordrecht.

Chen, M., and J. Tromp (2007). Theoretical and numerical investigation of global and regional seismic wave propagation in weakly anisotropic Earth models, Geophys. J. Int. 168, 1130-1152.

Chen, P., L. Zhao, and T. H. Jordan (2007). Full 3D tomography for the crustal structure of the Los Angeles region, Bull. Seismol. Soc. Am. 97, 1094-1120.

Chevrot, S. (2000). Multichannel analysis of shear wave splitting, $J$. Geophys. Res. 105, 21,579-21,590.

Chevrot, S. (2006). Finite-frequency vectorial tomography: a new method for high resolution imaging of mantle anisotropy, Geophys. J. Int. 165, 641-657.

Chevrot, S., N. Favier, and D. Komatitsch (2004). Shear wave splitting in three-dimensional anisotropic media, Geophys. J. Int. 159, 711-720.

Dahlen, F. A., S.-H. Hung, and G. Nolet (2000). Fréchet kernels for finitefrequency traveltimes-I. Theory, Geophys. J. Int. 141, 157-174.

de Hoop, M. V., R. D. van der Hilst, and P. Shen (2006). Wave-equation reflection tomography: annihilators and sensitivity kernels, Geophys. J. Int. 167, 1332-1352.

Dziewonski, A. M., and D. L. Anderson (1981). Preliminary reference Earth model, Phys. Earth Planet. Inter. 25, 297-356.

Favier, N., and S. Chevrot (2003). Sensitivity kernels for shear wave splitting in transverse isotropic media, Geophys. J. Inter. 153, 213-228.

Favier, N., S. Chevrot, and D. Komatitsch (2004). Near-field influence on shear wave splitting and traveltime sensitivity kernels, Geophys. J. Inter. 156, 467-482.

Hartog, R., and S. Y. Schwartz (2000). Subduction-induced strain in the upper mantle east of the Mendocino triple junction, California, $J$. Geophys. Res. 105, 7909-7930.

Hartog, R., and S. Y. Schwartz (2001). Depth-dependent mantle anisotropy below the San Andreas fault system: apparent splitting parameters and waveforms, J. Geophys. Res. 106, 4155-4167.

Hung, S.-H., F. A. Dahlen, and G. Nolet (2000). Fréchet kernels for finite-frequency traveltimes-II. Examples, Geophys. J. Int. 141, 175-203.

Kaminski, E., and N. L. M. Ribe (2002). Timescales for the evolution of seismic anisotropy in mantle flow, Geochem. Geophys. Geosyst. 3, no. 8, 1051, doi 10.1029/2001GC000222.

Komatitsch, D., and J. Tromp (1999). Introduction to the spectral element method for three-dimensional seismic wave propagation, Geophys. J. Int., 139, 806-822.

Komatitsch, D., and J. P. Vilotte (1998). The spectral-element method: an efficient tool to simulate the seismic response of 2D and 3D geological structures, Bull. Seismol. Soc. Am., 88, no. 2, 368-392.

Lev, E., M. D. Long, and R. D. van der Hilst (2006). Seismic anisotropy in Eastern Tibet from shear wave splitting reveals changes in lithospheric deformation, Earth Planet. Sci. Lett. 251, 293-304.

Levin, V., W. Menke, and J. Park (1999). Shear wave splitting in the Appalachians and the Urals: a case for multilayered anisotropy, $J$. Geophys. Res. 104, 17,975-17,993.

Liu, Q., and J. Tromp (2006). Finite-frequency kernels based upon adjoint methods, Bull. Seismol. Soc. Am. 96, 2383-2397. 
Long, M. D., and R. D. van der Hilst (2005). Estimating shear-wave splitting parameters from broadband recordings in Japan: a comparison of three methods, Bull. Seismol. Soc. Am. 95, 1346-1358.

Luo, Y., and G. T. Schuster (1991). Wave-equation traveltime inversion, Geophysics 56, 645-653.

Maggi, A., C. Tape, Q. Liu, M. Chen, and J. Tromp (2006). An automated waveform-similarity phase selection algorithm for fully $3 \mathrm{D}$ adjoint tomography (Abstract S51A-1250), Eos Trans. AGU 87, no. 52 (Fall Meet. Suppl.), S51A-1250.

Marquering, H., F. A. Dahlen, and G. Nolet (1999). Three-dimensional sensitivity kernels for finite-frequency travel-times: the banana-doughnut paradox, Geophys. J. Int. 137, 805-815.

Montagner, J. P., and H. C. Nataf (1986). A simple method for inverting the azimuthal anisotropy of surface waves, J. Geophys. Res. 91, $511-520$.

Montagner, J. P., D. A. Griot-Pommera, and J. Lavé (2000). How to relate body wave and surface wave anisotropy?, J. Geophys. Res. 105, 19,015-19,027.

Montelli, R., G. Nolet, G. Masters, F. A. Dahlen, and S.-H. Hung (2004), Global P and PP travel time tomography: rays versus waves, Geophys. J. Int. 158, 637-654.

Polet, J., and H. Kanamori (2002). Anisotropy beneath California: shear wave splitting measurements using a dense broadband array, Geophys. J. Int. 149, 313-327.

Ristema, J., and H.-J. van Heijst (2002). Constraints on the correlation of $P$ - and $S$-wave velocity heterogeneity in the mantle from P, PP, PPP and PKPab traveltimes, Geophys. J. Int. 149, 482-489.

Robinson, E. A., and S. Treitel (1980). Geophysical Signal Analysis, Prentice Hall, New York.

Sieminski, A., Q. Liu, J. Trampert, and J. Tromp (2007a). Finite-frequency sensitivity of surface waves to anisotropy based upon adjoint methods, Geophys. J. Int. 168, 1153-1174.

Sieminski, A., Q. Liu, J. Trampert, and J. Tromp (2007b). Finite-frequency sensitivity of body waves to anisotropy based upon adjoint methods, Geophys. J. Int. 171, 368-389.

Silver, P. G., and W. W. Chan (1988). Implications for continental structure and evolution from seismic anisotropy, Nature 335, 34-39.

Silver, P. G., and W. W. Chan (1991). Shear wave splitting and subcontinental mantle deformation, J. Geophys. Res. 96, 16,429-16,454.

Silver, P. G., and M. K. Savage (1994). The interpretation of shearwave splitting parameters in the presence of two anisotropic layers, Geophys. J. Int. 119, 949-963.
Smith, M. L., and F. A. Dahlen (1973). The azimuthal dependence of Love and Rayleigh wave propagation in a slightly anisotropic medium, J. Geophys. Res. 78, 3321-3333.

Su, W.-J., and A. M. Dziewonski (1992). On the scale of mantle heterogeneity, Phys. Earth Planet. Inter. 74, 29-54.

Tape, C., Q. Liu, and J. Tromp (2007). Finite frequency tomography using adjoint methods-methodology and examples using membrane surface waves, Geophys. J. Int. 168, 1105-1129.

Thomsen, L. (1986). Weak anisotropy, Geophysics 51, 1954-1966.

Tromp, J., C. Tape, and Q. Liu (2005). Seismic tomography, adjoint methods, time reversal and banana-doughnut kernels, Geophys. J. Int. 160, 195-216.

Vinnik, L. P., V. Farra, and Romanowicz B. (1989). Azimuthal anisotropy in the Earth from observations of $S K S$ at Geoscope and NARS broadband stations, Bull. Seismol. Soc. Am. 79, 1542-1558.

Woodward, R. L., and G. Masters (1991). Global upper mantle structure from ScS-S differential travel times, J. Geophys. Res. 96, 6351-6377.

Wüstefeld, A., and G. Bokelmann (2007). Null detection in shear-wave splitting measurements, Bull. Seismol. Soc. Am. 97, 1204-1211.

Zhao, L., and T. H. Jordan (2006). Structural sensitivities of finite-frequency seismic waves: a full-wave approach, Geophys. J. Int. 165, 981-990.

Zhao, L., T. H. Jordan, K. B. Olsen, and P. Chen (2005). Fréchet kernels for imaging regional Earth structure based on three-dimensional reference models, Bull. Seismol. Soc. Am. 95, 2066-2080.

Department of Earth Sciences

Utrecht University

P.O. Box 80021

TA 3058

Utrecht, The Netherlands (A.S., H.P., J.T.)

Seismological Laboratory

California Institute of Technology

Pasadena, California 91125

(J.T.) 